\title{
Transportation of sputum samples in cetylpyridinium chloride for drug resistance studies from remote areas of Odisha, India
}

\author{
Dasarathi Das, Bhagirathi Dwibedi, Shantanu Kumar Kar \\ Regional Medical Research Centre, Bhubaneswar, Odisha, India
}

\begin{abstract}
Introduction: Antimicrobial susceptibility testing of Mycobacterium tuberculosis is required for successful treatment of patients, mainly in retreatment cases which necessitate isolation of mycobacteria from sputum samples within 24-48 hours. In situations where transportation of sputum is required, the use of cetylpyridinium chloride (CPC) effectively sustains the viability of mycobacteria up to two weeks.

Methodology: Sputum samples were collected from pulmonary TB patients attending designated microscopy centres (DMC), stored in CPC solution and transported to a culture drug susceptibility testing laboratory using overnight bus transport facilities. For culture, the sputum specimens were processed and inoculated in Lowenstein- Jensen (LJ) medium. Growth on LJ was identified by colony morphology, growth rate and biochemical tests, and transit time was calculated as the time taken from the date of sample collection to the inoculation date.

Results: Out of the 816 sputum samples collected in CPC, 691 (84.7\%) yielded M. tuberculosis, 97 (11.9\%) yielded no growth, 21(2.6\%) grew contaminants and $7(0.8 \%)$ were nontuberculous mycobacteria. CPC containing sputum samples processed within two weeks showed $88.6 \%$ culture positivity, while positivity was significantly affected beyond two weeks.

Conclusions: CPC is cheap, easy to use, inhibits the growth of other organisms and can effectively be used to transport sputum specimens within two weeks from hard to reach areas to central locations without compromising culture positivity. Bus transport services can also help in reducing delay and the cost of transportation from remote areas.
\end{abstract}

Key words: CPC; Sputum transportation; remote areas.

J Infect Dev Ctries 2014; 8(8):1059-1062. doi:10.3855/jidc.4384

(Received 05 November 2013 - Accepted 23 January 2014)

Copyright (C) 2014 Das et al. This is an open-access article distributed under the Creative Commons Attribution License, which permits unrestricted use, distribution, and reproduction in any medium, provided the original work is properly cited.

\section{Introduction}

Tuberculosis is one of the major public health problems in many remote areas of low and middle income countries of the world. In such countries this situation is further challenged by the emergence of multi drug resistant (MDR) and extensively drug resistant (XDR) strains. For successful treatment of patients infected with tuberculosis and aiming at generating area-specific drug resistance patterns, drug susceptibility testing is being carried out in many remote parts of the globe, both in rural and urban areas. The processes involve transporting sputum samples for culture to specialized laboratories usually located in major towns. Studies have shown that, sputum specimens kept at room temperature beyond 3 days without preservatives, suffer a significantly low yield of isolation of Mycobacterium tuberculosis [1].

Only one study on drug resistance which used cetylpyridinium chloride (CPC) as a transport medium [2] has been previously conducted in the Mayurbhanj district, state of Odisha (India), that has hilly and forest covered areas similar to the area of Rayagada.

In India, CPC has been found to provide a good transport medium, and sample processing within a week is therefore strongly recommended [3-5]. Logistic problems related to transportation of sputum specimens from remote, hilly, forest areas have often contributed to delay and hence exposed $M$. tuberculosis culture specimens to the risk of being stored for a long period of time in CPC.

\section{Methodology}

This study was included into a cross-sectional study undertaken in collaboration with the state and district TB department belonging to the government of Odisha, between June 2011 and May 2013, for assessment of anti-TB drug resistance in Rayagada district. The district is located between $19^{\circ} 0^{\prime}$ and $19^{0} 58^{\prime}$ north latitude and $82^{\circ} 5^{\prime}$ and $84^{\circ} 2^{\prime}$ east longitude in the southern part of Odisha, with $43 \%$ of the region covered by forests. According to the 2001 
census, the district has a population of 830 thousand, living in 2,667 rural villages and five urban/towns Out of the 20 designated microscopy centres (DMC), five are placed in the most important towns. Except few areas, communication by public transport to the district interior is not frequent and in some areas it is scantly available and extremely complicated in rainy seasons.

Sputum samples used for this study were collected from pulmonary TB patients attending 18 out of 20 DMC's, as the number of patients enrolled in two DMC's was very low (less than 30 per year). The DMCs were supplied with freshly prepared $1 \%$ CPC in $2 \%$ Sodium chloride solution and replaced fortnightly with fresh provisions. Sputum samples were collected in sterile $50 \mathrm{~mL}$ Falcon tubes to which equal volumes of CPC were added, tightly capped, sealed, labeled and kept at room temperature in the DMC by the Revised National Tuberculosis Control Programme (RNTCP) laboratory technician . The sputum samples were usually collected within a week by the project staff posted at the Regional Medical Research Centre Field Unit (RMRC-FU) at the Rayagada district headquarter hospital (DHH), following a phone communication from the respective DMC laboratory technician. Samples were transported by the project staff from the DMC's to RMRC-FU by using the public transport system. At the RMRC-FU the sputum samples collected from various DMC's throughout the week were packed (triple packing) and transported weekly to Bhubaneswar city using an overnight public transport facility, preferably on Monday or Tuesday. Specimens were delivered by a person in charge from the bus service and processed on the same day at the Revised National Tuberculosis Control Programme approved Tuberculosis Culture and Drug Susceptibility Testing Laboratory of RMRC in Bhubaneswar, based on the standard method [3].

For culture, the specimens were centrifuged at
$3000 \times \mathrm{g}$ for 15 minutes; sediment was re-suspended again in about $40-45 \mathrm{ml}$ sterile distilled water and centrifuged at $3000 \times \mathrm{g}$ for 15 minutes. Sediment was resuspended in 1-2 $\mathrm{ml}$ of sterile distilled water and a loopful (10ul) was inoculated in Lowenstein- Jensen (LJ) medium. The medium bottles were incubated at $37^{\circ} \mathrm{C}$ in a walk-in-incubator and were examined for growth of $M$. tuberculosis once per week, up to 8 weeks. The growth of $M$. tuberculosis complex was graded as follows: actual number of colonies if between 1 and 19 colonies; $1+$ for growth of $>20$ but $<100$ colonies; $2+$ for growth of $>100$ colonies; and $3+$ for confluent growth. Growth on LJ was identified by colony morphology, growth rate and biochemical tests, including niacin production, catalase activity at $68^{\circ} \mathrm{C}$ and susceptibility to p-nitrobenzoic acid. The transit time was calculated as the time taken from the date of sample collection to the inoculation date. Written informed consent was obtained from all patients. The Ethical Committee of RMRC, Bhubaneswar approved the study protocol.

\section{Results}

Out of 816 sputum samples collected in CPC, 691 (84.7\%) yielded M. tuberculosis, 97 (11.9\%) yielded no growth, $21(2.6 \%)$ grew contaminants and $7(0.8 \%)$ were nontuberculous mycobacteria. It was observed that $43.0 \%, 69.1 \%$ and $84.3 \%$ of sputum samples were processed within the first, second and third week respectively (Table 1). About $30.9 \%$ of sputum specimens could not be processed within two weeks, as transport took a longer time. The isolation of positive culture was not statistically significant among sputum samples processed within 2 weeks. It was observed that beyond 2 weeks the culture positivity was significantly affected $(p<0.05)$ in comparison to samples processed up to two weeks, while there was no effect on contamination rate. Specimens processed beyond 3 weeks were considerably affected $(\mathrm{p}<$

Table 1. Effect of CPC on isolation of M. tuberculosis in LJ medium.

\begin{tabular}{|c|c|c|c|c|}
\hline $\begin{array}{c}\text { Number of sputum } \\
\text { samples(Processed } \\
\text { within days) } \\
\end{array}$ & $\begin{array}{c}\text { Culture positive } \\
\text { N }(\%)\end{array}$ & $\begin{array}{c}\text { Culture negative } \\
\text { N (\%) }\end{array}$ & $\begin{array}{c}\text { Contamination } \\
\text { N (\%) }\end{array}$ & $\begin{array}{l}\text { NTM } \\
\text { N (\%) }\end{array}$ \\
\hline $351(1-7)$ & $311(88.6 \%)$ & $34(9.6 \%)$ & $6(1.7 \%)$ & $0(0 \%)$ \\
\hline $124(15-21)$ & $101(81.4 \%)$ & $16(12.9 \%)$ & $5(4.0 \%)$ & $2(1.6 \%)$ \\
\hline $64(22-28)$ & $45(70.3 \%)$ & $16(25.0 \%)$ & $2(3.1 \%)$ & $1(1.6 \%)$ \\
\hline $34(29-35)$ & $25(73.5 \%)$ & $8(23.5 \%)$ & $0(0 \%)$ & $1(2.9 \%)$ \\
\hline $6(43-49)$ & $4(66.7 \%)$ & $2(33.3 \%)$ & $0(0 \%)$ & $0(0 \%)$ \\
\hline $4(50-56)$ & $2(50.0 \%)$ & $2(50.0 \%)$ & $0(0 \%)$ & $0(0 \%)$ \\
\hline Total & $691(84.7 \%)$ & $97(11.9 \%)$ & $21(2.6 \%)$ & $7(0.8 \%)$ \\
\hline
\end{tabular}

CPC-Cetylpyridinium chloride, LJ- Lowenstein-Jensen, NTM- Non tuberculous mycobacteria 
0.0001) in comparison to two weeks exposure to CPC. Smear grade was compared with culture result and specimens with negative or scanty smear grade yielded significantly more negative cultures $(\mathrm{p}<0.001)$ than smears with $1+, 2+$ or $3+$ grades (Table 2 ). The growth of colonies in LJ medium was dependent on smear grade and significantly less confluent growth occurred in sputum samples incubated up to 8 weeks with negative or scanty acid fast bacilli (AFB).

\section{Discussion}

The primary requirement for a solid culture-based drug susceptibility testing is the need of a sputum sample free from contamination by other organisms than mycobacterium and its confluent growth in LJ media. We noted lower contamination rate $(<5 \%)$ from samples processed up to 6 weeks (samples processed beyond 6 weeks were few in number) from collection. This may be due to quick addition of CPC to the sputa by the laboratory technician as the patients were already found positive by Ziehl Neelsen microscopy. Previous studies had shown that CPC kills pathogenic fungi from sputum specimens [6] and reduce the contamination rate in sputa cultured in solid media [3]. The earlier study in Odisha reported a contamination rate of $9.9 \%$ and attributed it to noncompliance of immediate CPC addition and transport delays [2]. In comparison to earlier studies, these results show an overall culture positivity of $84.7 \%$. However, the samples tested within two weeks of storage in CPC showed $88.6 \%$ culture positivity. The culture positivity observed beyond three weeks was comparable and in agreement with earlier reports in which samples were processed after 20 days or longer [4]. While processing the sputum samples it was noted that single centrifugation as recommended $[7,8]$ was not able to remove traces of CPC and it caused a deepening at the site of inoculation on LJ slants. We suggest a further wash with sterile distilled water for good colonies of $M$. tuberculosis on LJ slants, as reported by a previous study [9]. The culture negativity was significantly higher $(\mathrm{p}<0.001)$ in negative and scanty smears in comparison to smears with $1+$ or more AFB grades. Out of 691 culture positives, $81.5 \%$ specimens showed confluent growth within eight weeks of incubation (Table 2). It was observed that a higher number of sputum specimens with $1+$ or higher AFB grades showed confluent growth within eight weeks compared to scanty and negative smear specimens. During our study, sputum specimens were collected both from newly diagnosed and previously treated cases with anti TB treatment and not differentiated, as CPC has no effect on isolation of mycobacteria [10]. We used public bus services to transport pooled sputum samples from Rayagada district to RMRC, the Bhubaneswar laboratory situated at a distance of $500 \mathrm{kms}$ and found that this is effective in reducing transportation costs, human resources requirements and delay in delivery, as previously reported for difficult areas [11].

\section{Conclusion}

The study showed that using public bus services to transport sputum from remote, hilly, forest and hard to reach areas located as far as $500 \mathrm{kms}$ away from testing point was effective in reducing transportation costs, human resources requirements and minimized delays pertaining to delivery of specimen. In addition, CPC is inexpensive, easy to use, inhibits the growth of other organisms and can effectively be used to transport sputum specimens within two weeks from hard to reach areas to central locations, without compromising culture positivity. This may therefore be a possible alternative and an appropriate solution in most resource-constrained countries where longdistance transportation of sputum specimen for drug susceptibility testing is required.

Other low income countries, having geographical limitations such as rural location, remoteness, hilly and forest covered areas or hard to reach and/or underserved communities, may use the method described in this study to improve effectiveness of

Table 2. Comparison of smear grade with culture negativity and growth rate up to 8 weeks.

\begin{tabular}{ccc}
\hline $\begin{array}{c}\text { AFB Smear grade } \\
\text { (Number of samples) }\end{array}$ & $\begin{array}{c}\text { Culture negative } \\
\text { N (\%) }\end{array}$ & $\begin{array}{c}\text { Confluent growth observed up to 8 } \\
\text { weeks out of culture positives } \\
\text { N (\%) }\end{array}$ \\
\hline Negative (28) & $7(25 \%)$ & $7(33.3 \%)$ \\
Scanty (46) & $14(30.4 \%)$ & $23(71.9 \%)$ \\
$1+(343)$ & $41(12 \%)$ & $230(79.6 \%)$ \\
3+ (217) & $20(9.2 \%)$ & $162(86.6 \%)$ \\
Total & $15(8.2 \%)$ & $141(87.0 \%)$ \\
\hline
\end{tabular}

AFB-Acid fast bacilli, *AFB grade could not be evaluated for 9 samples 
delivering sputum and at the same time to reduce risk of damage related to isolation of Mycobacteria. There may be the need to make CPC-sodium chloride solution always available in such areas so that the quality of sputum is ensured and the risk to jeopardize culture positivity reduced.

\section{Acknowledgements}

We thank the State Tuberculosis Officer, District Tuberculosis Officer who permitted the collection of sputum samples for this study and also the laboratory technicians of Rayagada DMCs for specimen and information collection and RMRC Bhubaneswar laboratory for sputum processing for culture and drug susceptibility testing. This work was supported by the Indian Council of Medical Research under extramural research grant.

\section{References}

1. Paramsivan CN, Narayan ASL, Prabhakar R, Rajagopal MS, Somasundarm P, Tripathy SP (1983) Effect of storage of sputum specimens at room temperature on smear and culture results. Tubeivle 64: 119-124.

2. Mahadeo B, Kumar P, Agrawal SP, Chauhan LS, Srikantaramu N (2005) Surveillance of drug resistant to antituberculosis drugs in districts of Hoogli in West Bengal and Myurbhanj in Orissa. Indian J Tuberc 52: 5-10.

3. Smithwick RW, Stratigos CB, David HL (1975) Use of cetylpyridinium chloride and sodium chloride for the decontamination of sputum specimens that are transported to the laboratory for the isolation of Mycobacterium tuberculosis. J Clin Microbiol 1: 411-413.

4. Selvakumar N, Kumar V, Gopi PG, Venkataramu KV, Datta M, Paramasivan CN, Prabhakar R (1995) Isolation of tubercle bacilli from sputum samples of patients in the field studies by the cetylpyridinium chloride-sodium chloride \& sodium hydroxide methods. Ind J Med Res 102: 149-151.
5. Pardini M, Varaine F, Iona E, Arzumanian E, Checchi F, Oggioni MR, Orefici G, Fattorini L (2005) Cetyl-pyridinium chloride is useful for isolation of Mycobacterium tuberculosis from sputa subjected to long-term storage. J Clin Microbiol 43: 442-444.

6. Phillips BJ, Kaplan W (1976) Effect of cetylpyridinium chloride on pathogenic fungi and Nocardia asteroides in sputum. J Clin Microbiol 3: 272-276.

7. Aparna S, Krishnamoorthy KV, Gokhale S (2006) From microscopy centre to culture laboratory: a viable ride for mycobacteria. Int J Tuberc Lung Dis 10: 447-449.

8. Pal N, Sharma B, Malhotra B, Rishi S (2009) Transport and storage of sputum specimen by using cetylpyridinium chloride for isolation of mycobacteria. Ind J Pathol Microbiol 52: 59-61.

9. Selvakumar N, Kumar V, Narayana ASL, Suryanarayana D, Umapathy K (1993) Use of cetylpyridinium chloride for storage of sputum specimens and isolation of M. tuberculosis. Ind J Tuberc 40: 95-97.

10. Abraham PR, Kasetty SM, Sharma VD, Shivannavar CT(2012) Similarity in the isolation rate of Mycobacterium tuberculosis for new and treated cases of tuberculosis in sputum specimens preserved under cetylpyridinium chloride. J Glob Infect Dis 4: 90-91.

11. Harries AD, Michongwe J, Nyirenda TE, Kemp JR, Squire SB, Ramsay AR, Godfrey-Faussett P, Salaniponi FM (2004) Using a bus service for transporting sputum specimens to the Central Reference Laboratory: Effect on the routine TB culture service in Malawi. Int J Tuberc Lung Dis 8:204-210.

\section{Corresponding author}

Shantanu Kumar Kar

Regional Medical Research Centre, Bhubaneswar-751023, Odisha, India

Phone: 91-674-2301322

Fax: 91-674-2301351

Email: skk@icmr.org.in

Conflict of interests: No conflict of interests is declared. 